\title{
REVIEW
}

\section{Original New Theory Abbreviation of Metals}

\section{Senchun Jiang*}

Hangyu life saving Co., Ltd., Xiangyang, Hubei, 441002, China

\begin{tabular}{l}
\hline ARTICLE INFO \\
\hline Article history \\
Received: 9 September 2019 \\
Accepted: 10 September 2019 \\
Published Online: 31 October 2020 \\
\hline Keywords: \\
Gaussian curve \\
C-curve \\
Dispersion center \\
Supercooling dynamics \\
Carbon atom interaction
\end{tabular}

\section{Properties of Metallic Iron Atoms}

$\mathrm{T}$ The spatial arrangement of iron atoms changes according to temperature, lt is a body-centered cubic lattice at high temperature. The middle temperature is a face-centered cubic lattice, $1 \mathrm{t}$ is a body-centered cubic lattice at room temperature, The volume of ferrite cell is the largest at middle temperature, high temperature comes next, room temperature is the smallest. The high temperature BCC lattice structure is suitable for high temperature hot gas flow, medium-temperature face-centered cubic lattice structure fixed hot gas flow, good heat preservation performance, at room temperature, the $\mathrm{BCC}$ lattice structure is suitable for low temperature hot gas flow.

\section{Atomic Arrangement}

Regular arrangement of iron atoms does not allow other atoms to penetrate into the regular arrangement of the

*Corresponding Author:

Senchun Jiang,

Hangyu life saving Co., Ltd., Xiangyang, Hubei, 441002, China;

Email:2126828166@qq.com

\section{ABSTRACT}

The electrons of metallic iron atoms all have wave-particle duality. The electrons of each iron atom are particles in the liquid state, the solid state is fluctuating. The wave-particle duality parameters of each iron atom electron are different, there is a small difference, the same is gathered into one, become a single grain. The parameters of wave-particle duality are different. The crystallization temperature of liquid state is different from that of solid state $^{[1]}$.

gap, other atoms can only penetrate into the defects in the arrangement of iron atoms, size and distribution of point, line and surface defects, determine the quality of the metal iron. The number of point, line and area defects is limited, a finite quantity determines the finite carbon content of iron. The size of grain boundary space is limited.

When there are few carbon atoms at the grain boundary, the iron atoms deprived of iron grains are combined into cementite at the grain boundary, over time carbon atoms aggregate at the grain boundaries to form graphite.

\section{Iron Carbon Balance Diagram}

The carbon atoms keep the iron crystal structure unchanged, that is to say, the stability of Iron structure is increased. The lines A, H, J and B of the iron-carbon equilibrium diagram are the lines of crystal-structure transition, G, P, S are also lattice structure transition lines, these 
two lines show that: the carbon atoms remain unchanged in the original structure. Carbon atoms are enriched into the crystal defect space to maintain the stability of the crystal structure.

The grain volume and defect space volume change with temperature, causing the separation and absorption of carbon atoms change of temperature transforms the structure. The precipitated carbon atoms are then enriched into the space with large defects in other grains, grain growth is a process of volume change and lattice defects constantly precipitating and absorbing carbon atoms, until the structural transformation.

C curve is the Gaussian curve in statistics,analyzing the $\mathrm{C}$ curve with statistical theory and everything will be clear. The undercooling power causes the transformation of the grain structure to a lower temperature, the martensite with transition structure from FCC structure to bcc structure was obtained by quenching, this is due to the combined action of carbon atoms and supercooling force, which reduces the temperature of structural transformation, the transformation process becomes longer, the result of slowing down. The reason for the high hardness of martensite is that there are three iron atoms in the cell structure. lt's the structure that causes the hardness. The volume of martensite unit cell is the largest, as result, the lattice defect space is large, the carbon-containing ability is much greater than that of the defects in the face-cen- tered and body-centered lattices ${ }^{[2]}$.

The primary and secondary temper embrittlement is the reason why the crystal structure is unstable before and after the transformation, which leads to increased brittleness. Tempering does not change the lattice from BCC structure to marten site structure.

Alloy steel, carbon steel, cast iron, whether in solid or liquid, there is an objective fact of lattice structure transformation at $1493^{\circ} \mathrm{C}$.

The principles of materials science are: structure determines performance.we should understand the metal iron from the structure. The face-centered cubic structure does not conduct magnetism, the magnetic conductivity of body-centered cubic junction is the reason for the size and size of the structural gap. It is not the electrons of the iron atom that determine magnetic conductivity

\section{References}

[1] People's education press. Institute of curriculum and teaching materials. Edited by chemistry curriculum research center. Chemistry (compulsory 1). People's education press, 2004 preliminary examination and approval.

[2] Higher education press. Beijing Normal University. Central China Normal University. Nanjing Normal University. Inorganic chemistry. Volume 2. Fourth edition. 